\section{Environmental Contamination Sources and Control in High Resolution Scanning Electron Microscopy}

\author{
Ronald Vane and Vince Carlino \\ XEI Scientific, Redwood City, CA \\ Rvane@evactron.com
}

Traditionally, contamination control in SEMs has focused on pump oils, finger prints, dirty specimens, and good vacuum practice in manufacturing. Now, the use of dry pumps at all stages of the vacuum system of new FE SEMs, and the use of better vacuum practices on the part on users and manufacturers have made environmental hydrocarbons, the hydrocarbon background contamination of our world, a significant source of the remaining hydrocarbons in electron microscope vacuum systems. These environment sources of hydrocarbons (HC) cause a loss of resolution and contrast in imaging at the highest levels of magnification.

The semiconductor industry and associated nano-sciences have brought on a demand for instruments that can image structures $<5 \mathrm{~nm}$ in size at $<2 \mathrm{KV}$. Instrument manufacturers have responded with Field Emission (FE) instruments that can give better than 200KX magnification with ease and produce high contrast with low KV beams. Control of contamination has taken more importance as semiconductor manufacturers move to ever smaller dimensions. It is already common to be looking with low $\mathrm{KV}(<2 \mathrm{KV})$ at features $<10 \mathrm{~nm}$ in size that are close to the resolution limits of the instruments. In such cases, the smallest amount of $\mathrm{HC}$ in the chamber can cause a loss of resolution and contrast. The electron beam reacts with any stray $\mathrm{HC}$ in the beam path or on the surface to create $\mathrm{HC}$ ions that then condense and form a hydrocarbon gunk on the area being scanned. Despite dry pumps and LN traps, these artifacts and contamination haze continue to be formed. What is the source and what can be done?

It is important to understand that SEMs are not designed from the ground up as ultra high vacuum instruments. Unlike XPS and Auger spectrometer systems that require ultra high vacuum to obtain high resolution energy information from the emitted electrons, electron microscopes only look at electron flux. Backscatter detectors do have some energy discrimination, but the energy filtering is crude and does not need ultra high vacuum. High vacuum SEMs are not bothered by water vapor and can do imaging with pressures in the low $10^{-5}$ Torr range in the specimen chamber. But good SEM imaging is disrupted by HC. Roughing pump oil in the chamber with a partial pressure of $10^{-7}$ Torr will cause black squares to form on specimen images.

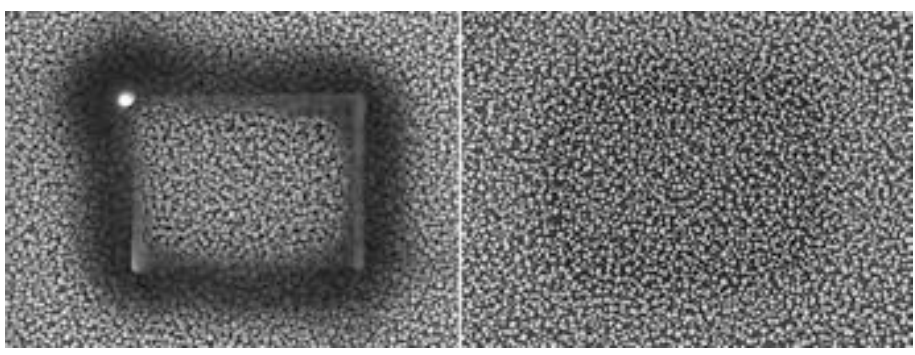

Figure 1. Two locations of a silicon "grass" sample irradiated for 10 minutes before (left) and after (right) the use of Evactron anticontamination device. 50KX. [1]

Exposure of samples and surfaces in a vacuum chamber to room air is an under-recognized source of environment $\mathrm{HC}$ contamination in microscopy. Long known and understood in the surface analysis, HC collects on all surfaces exposed to room air. Room air is full of trace amounts of organic vapors from plant, animal, and synthetic sources. XPS and Auger spectroscopy reveal a carbon background on everything exposed to it. The effective ways to rid surfaces of these carbon compounds are to drive them off or destroy them with heat, sputtering, or a chemical etch. Solvent cleaning may leave residues. A freshly prepared specimen will show little if any contamination, but the same sample left exposed to room air overnight or longer, even in a protective container, will show surface carbon build up.

Environmental $\mathrm{HC}$ can also enter into an EM chamber whenever the chamber is opened for specimen exchange or instrument servicing. Servicing the instrument for stage repairs, detector exchange, pole piece replacement, etc. can bring in HC from room air. During servicing, cleaning parts with solvents is the traditional method, the results are uncertain until the system is pumped down and an image taken.

Environmental contamination will also accumulate on specimens used for system performance tests. The common gold on Carbon specimens are unusually contaminated by exposure to environmental $\mathrm{HC}$ inside their carrier cases. These specimens are best when plasma cleaned before use.

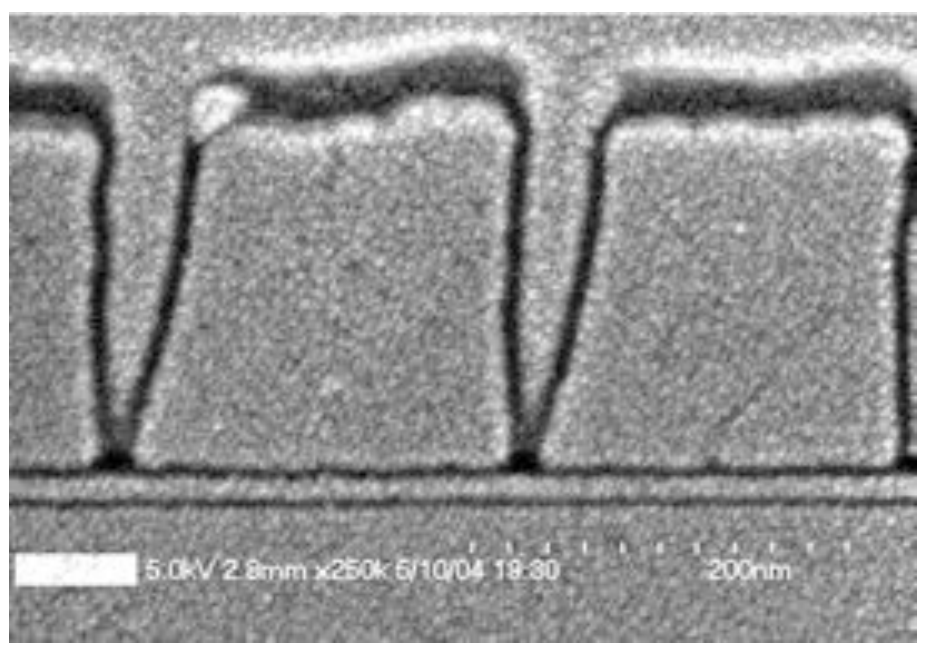

Figure 2. Example of resolution improvement after Evactron cleaning. Small sputter Pt islands are visible on a semiconductor cross section at 250 KX at $5 \mathrm{KV}$ on a 6 year old Hitachi $S 4700$.

Plasma cleaning of specimens before they are placed in the EM chamber is a commonly applied method to stop contamination from reaching the EM chamber. Desk top plasma cleaners for cleaning specimens and TEM stages are available from several manufacturers. These cleaners use exposure to argon, oxygen, or mixed gas plasmas to sputter etch the HC layer off the surface of the specimen and stage. Used for too long they cause sputter damage to the specimen surfaces.

The XEI Scientific Evactron Anti-Contaminator offers an alternate cleaning method that removes environmental $\mathrm{HC}$ and $\mathrm{HC}$ from any other source from the inside of electron microscopes and dual beam FIBs. Using remote RF plasma to make $\mathrm{O}$ radicals from air, Evactron cleaning uses the pumping differential to flow the oxygen radicals though the specimen chamber and oxidize off the $\mathrm{HC}$ from all surfaces. The process is quick to remove the typical low levels of $\mathrm{HC}$ present, and it possible to complete cleaning in less than 5 minutes on many specimens and chambers.

\section{References:}

[1] András E. Vladár, Michael T. Postek and Ronald Vane* "Active Monitoring and Control of Electron Beam Induced Contamination" Proc. SPIE Vol. 4344 (2001), 835. 


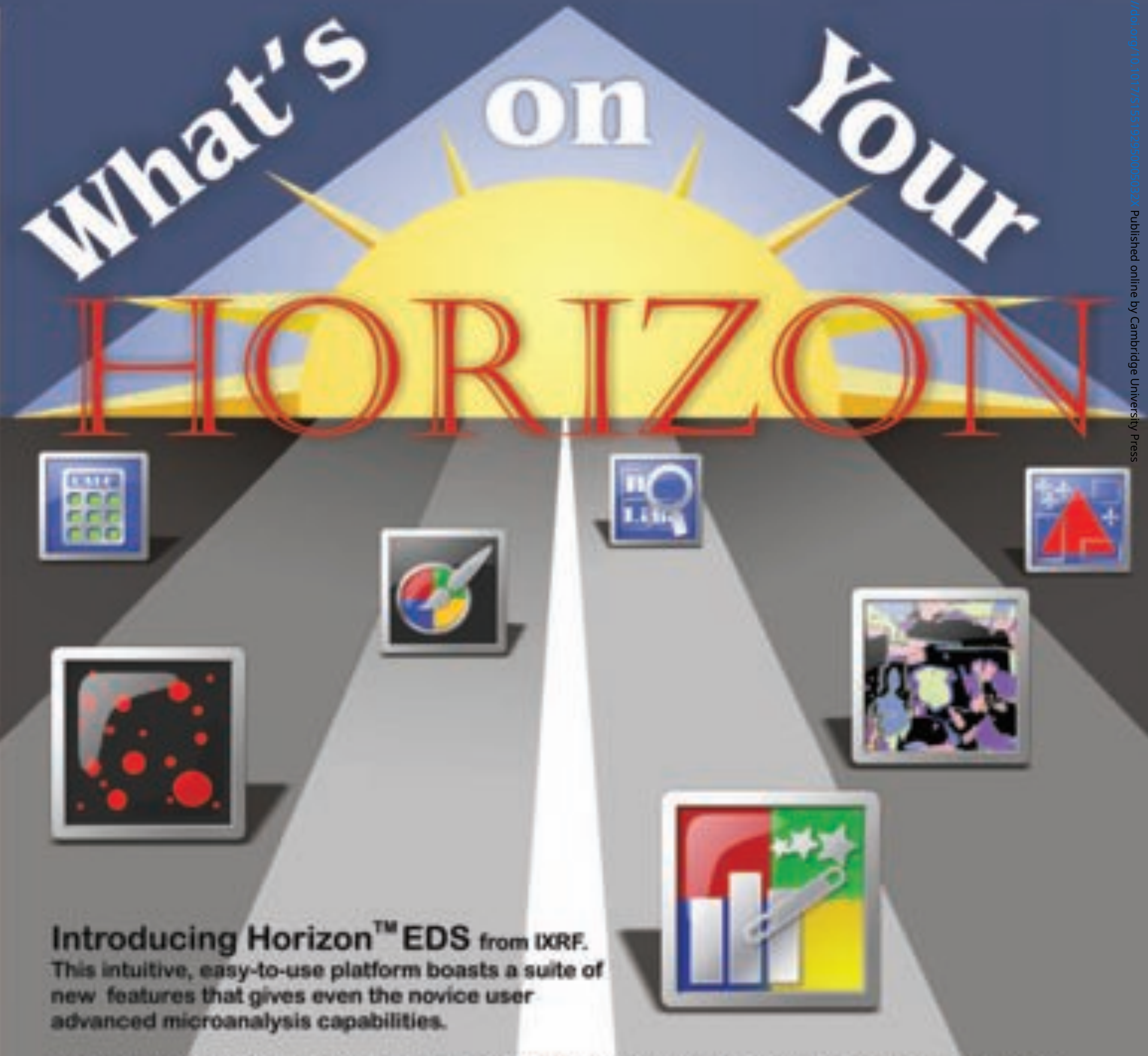

Horizon" EDS packages come standard with FREE software upgrades for life and a myriad of high-end options that are typically costly add-ons from the other vendors.

Stop by our booth $\$ 1414$ to see what might be on your HORIZON!

IXRF customers enjoy:

- FREE Software for Life

* 3yr Warranty (including detector)

* Premium $\leq 130 \mathrm{eV}$ Detectors

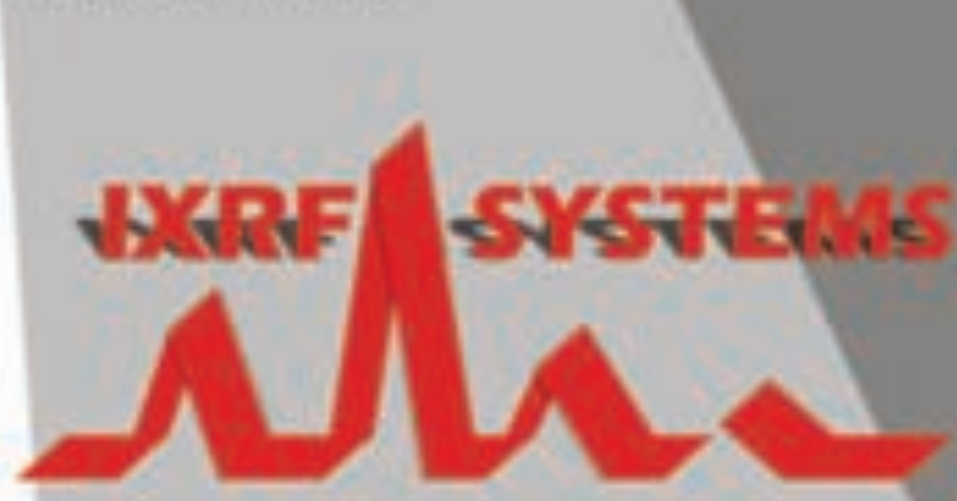

